\title{
A SYMBOLIC TREATMENT OF THE GEOMETRY OF HYPERSPACE*
}

BY

\author{
LOUIS INGOLD
}

\section{INTRODUCTION}

The beautiful symbolic method of Maschket for the study of differential invariants has been applied to a certain extent to the study of geometry. Maschke himself has made application to the theory of curvature of hyperspace and also to the study of directional relations.t The more important formulas of ordinary differential geometry were developed by Smith $\S$ in terms of the symbolic notations, and finally, Bates" has used the method in a further study of curvature.

Of these applications, the nost complete and satisfactory is that of Maschke in his paper Differential parameters of the first order. This paper contains a complete discussion of the relations between the tangent vectors to the subspaces $R_{k}$ of the space $S_{n}$ under consideration.

The development of those properties of hyperspace which depend upon invariants and differential parameters of the second order is by no means as complete. Several of the papers mentioned above are, to be sure, devoted to special problems involving second order properties, but no systematic study of these properties by means of the symbolic method has been attempted.

There is, of course, a very extensive development of this subject by means of unsymbolic methods. A quite complete treatment of the geometry of two-dimensional surfaces has been given by Wilson and Mooreף and

\footnotetext{
* Presented to the Society (Southwestern Section), December 1, 1923.

$\uparrow$ Maschke, A new method of determining the differential parameters and invariants of quadraicic differential quantics, these Transactions, vol. 1 (1900), pp. 197-204, and A symbolic treatment of the theory of invariants of quadratic differential quantics in $n$ variables, ibid., vol. 4 (1903), pp. 445-469. This paper will be referred to as Invariants.

$\ddagger$ Maschke, The Kronecker-Gaussian curvature of hyperspace, and Differential parameters of the first order, these Transactions, vol. 7 (1906), pp. 69-80, and pp. 81-93.

$\S \mathrm{A}$. W. Smith, The symbolic treatment of differential geometry, these Transactions, vol. 7 (1906), pp. 33-60.

II Bates, An application of symbolic methods to the treatment of mean curvatures in hyperspace, these Transactions, vol. 12 (1911), pp. 19-38.

Tा E. B. Wilson and C. L. E. Moore, Differential geometry of two-dimensional surfaces in hyperspace, Proceedings of the American Academy of Arts and Sciences, vol. 52 (1916), p. 269.
} 
Moore* has extended some of their results to spaces of $n$ dimensions. These authors make use of the method of the absolute differential calculus, $\dagger$ and this method has been employed in the majority of recent papers on this subject.

It is the object of this paper to study the second order properties of $n$-dimensional manifolds by means of the symbolic method of Maschke. It is found that many important results and formulas of the geometry of hyperspace are easily derived by this method. One advantage of the method is the ease with which relations may be derived connecting various invariants and differential parameters.

An advantage of the method of the absolute calculus which is always, very justly, insisted upon is that derived from the fact that invariant expressions are immediately recognizable on account of their form. This applies with equal force to the symbolic method. It may be added that a general vector interpretation of the Maschke symbol makes it possible to study the properties of hyperspace without reference to a containing euclidean space.

Incidentally the paper contains a number of extensions of the symbolic theory: for example, a formula is found which expresses as an invariant, or differential parameter, the derivative of any function of the coördinates with respect to arc length along any curve.

By means of this formula it is possible not only to obtain a large number of invariants but also, from known relations connecting differential parameters, to obtain still other relations. The formula also aids very materially in the geometric interpretation of invariant forms.

In order to avoid frequent interruptions in the geometric applications a general account of the notations and interpretations of the symbolic theory has been given in Part I. This part also includes certain general formulas that are of frequent occurrence in the latter portions of the paper. Part II treats the geometry of two-dimensional manifolds in considerable detail. In Part III is given an outline of the extension to $n$ dimensions.

\section{Notations AND general Formulas}

1. First fundamental form. If the space $S_{n}$ under consideration is contained in a euclidean space we may let $f$ denote the vector from an arbitrary origin to the points of $S_{n}$. The square of the differential of arc length along any curve of $S_{n}$ is then given by

$$
\imath s^{2}=\left(\sum f_{i} d u_{i}\right)^{2}=\sum f_{i} f_{j} d u_{i} d u_{j}=\sum E_{i j} d u_{i} d u_{j},
$$

${ }^{*}$ C. L. E. Moore, Note on minimal varieties in hyperspace, Bulletin of the American Mathematical Society, vol. 27 (1921), p. 216.

$\dagger$ For references see the paper by Wilson and Moore, loc. cit. 
where the multiplication of the vectors is the inner or scalar multiplication, and the subscript $i$ denotes differentiation with respect to the corresponding variable $u_{i}$.

The scalar products $f_{i} f_{j}$ are the coefficients of the differential form (1). The vector $f$, therefore, has precisely the properties of Maschke's symbolic function belonging to (1). $t$

It is not necessary, however, to assume that $S_{n}$ is contained in a euclidean space. The developments of the paper apply to spaces $S_{n}$ defined, or characterized, by a function $f\left(x ; u_{1}, u_{z}, \ldots, u_{n}\right)$ which is such that

$$
\int_{a}^{b} f_{i} f_{j} d x=E_{i j}
$$

There are, of course, functions of this sort that are not expressible by means of a finite number of terms in the form

$$
\sum_{i} U_{i} e_{i}(x)
$$

- where the coefficients $U_{i}$ are functions of the parameters $u_{i}$ only. For many such interpretations of the symbol $f$ there can be obtained a theory wholly analogous to the geometric theory.

In order to form expressions of higher than the first degree in the coefficients $E_{i j}$ Maschke introduced other symbols $\varphi, \psi$, or $f^{1}, f^{2}, \ldots, \varphi^{1}, \varphi^{2}, \ldots$, equivalent to the symbol $f^{*}$ (Upper indices will be frequently used instead of different letters to indicate distinct functions or symbols. Exponents are seldom used and when used will be easily recognized.) For the purposes of this paper, these will be regarded as equivalent notations for the vector $f$; thus, $f_{1} f_{2} \varphi_{1} \varphi_{2}=E_{12}^{2}$ and $f_{1} f_{2} \varphi_{2} \varphi_{3} \psi_{1} \psi_{3}=E_{12} E_{23} E_{31}$.

Those symbols (or notations) that occur precisely twice in a symbolic product are to be combined as scalar products. If there is only one symbol that occurs just once, the expression represents a simple vector. The same symbol (or notation) never occurs more than twice as a factor. It is very useful to notice that if each of two distinct symbols occurs precisely twice as a factor, then the two symbols may be interchanged without affecting the value of the product.

${ }^{*}$ The subscript is generally used in this sense, but occasionally it is used merely as a distinguishing mark, as for example in the fundamental quantities $\boldsymbol{E}_{i j}$.

$\dagger$ For details relating to the vector interpretation of symbolic forms see the author's paper Vector interpretation of symbolic differential parameters, these Transactions, vol. 11 (1910), pp. 449-474; also Functional differential geometry, ibid., vol. 13 (1912), pp. 318-341. 
Maschke has shown that if $F^{1}, F^{2}, \ldots, F^{n}$ are $n$ arbitrary functions of the variables $u_{1}, \cdots, u_{n}$, or any invariants of the form (1), then $\beta$ times the jacobian of $F^{1}, \ldots, F^{n}$ is again an invariant of (1), where $\beta$ denotes the reciprocal square root of the determinant $\left|E_{i j}\right|$. He calls this product

$$
\beta\left\{F^{1}, F^{2}, \cdots, F^{n}\right\}
$$

an invariantive constituent of the form (1) and denotes it by $\left(F^{1}, F^{2}, \ldots, F^{n}\right)$ or more briefly by $(F) .^{*}$

2. Vector invariants of the first order. In the invariantive constituent just described some or all of the F's may be symbols or other invariantive constituents. If $k$ distinct symbols occur just once the invariantive constituent represents a $k$-dimensional vector; thus $(\varphi a)=\left(\varphi^{1}, \cdots, \varphi^{k}, a^{1}, \cdots, a^{n-k}\right)+$ represents a $k$-dimensional vector tangent to the $k$-dimensional subspace determined by the equations $a=$ const., $a=$ const., $, \cdots, a=$ const. This vector is clearly invariant under change of parameters.

Other important invariants may be obtained by multiplying $(\varphi a)$ by a constituent which contains one or more of the notations $\varphi$ together with other symbols or scalar functions. In this way is formed the invariant vector $(f \varphi)(\varphi a)=\left(f^{1} \cdots f^{n-k} \varphi^{1} \cdots \varphi^{k}\right)\left(\varphi^{1} \cdots \varphi^{k} a^{1} \cdots a^{n-k}\right)$ which represents an $(n-k)$-dimensional vector tangent to $S_{n}$ but normal to the space determined by the $a^{i}$ as indicated above.

In some cases the product of several invariantive constituents may be interpreted in more than one way; thus the identity

$$
\left(f V^{1} \cdots V^{r-1} \varphi^{1} \cdots \varphi^{n-r}\right)\left(u^{1} \cdots u^{r} \varphi^{1} \cdots \varphi^{n-r}\right)\left(f u^{1} \cdots u^{r} w^{1} \cdots w^{n-r}\right)=0
$$

may be interpreted as stating that the vector $(f V \varphi)(u \varphi)$ is orthogonal to the vector $(f u w)$ or that the two $(n-r)$-dimensional vectors $(f V \varphi)(f u w)$ and $(u \varphi)$ are orthogonal to each other. In general the expression

$$
\left(f V^{1} \ldots V^{r-1} \varphi^{1} \cdots \varphi^{n-r}\right)\left(f a^{1} \cdots a^{n-1}\right)
$$

represents an $(n-r)$-dimensional vector tangent to $S_{n}$ but normal to the subspace determined by the equations

\footnotetext{
* Maschke, (Invariants, loc. cit., p. 447) uses the brackets \{\} to denote the jacobian of the quantities enclosed and reserves the parenthesis ( ) for $\beta$ times the jacobian.

$\dagger$ Maschke writes this in the form $\left(\varphi^{1}, \cdots, \Phi^{k}, a\right)$ where $a$ represents $a^{1}, a^{2}, \cdots, a^{n-k}$. See Invariants, loc. cit., p. 448,. Where no misunderstanding is likely to occur this may be further condensed to $(\phi a)$.
} 


$$
a^{i_{1}}=\text { const., } a^{i_{2}}=\text { const. }, \cdots, a^{i_{r}}=\text { const. }
$$

where $i_{1}, i_{2}, \cdots, i_{r}$ is any combination of $r$ of the indices $1,2, \cdots, n-1$.

3. Vector operators. It is sometimes convenient to regard symbolic invariantive constituents as vector operators; thus in any of the products of the preceding article either factor could be regarded as an operator applied to the other factor. The operator $\left(f^{1} \cdots f^{k} \varphi^{1} \cdots \varphi^{n-k}\right)$ applied to the $k$-dimensional vector $\left(f^{1} \cdots f^{k} a^{1} \cdots a^{n-k}\right)$ converts it into an $(n-k)$ dimensional vector in a space normal to the operand. This operator is thus analogous to Grassmann's operation of taking the supplement of a vector.

There are linear combinations of invariantive constituents which cannot be regarded as simple vectors, but these can often be regarded as compound elements in Grassmann's sense, or they may be regarded as tensors; for example, the sum $(f \varphi a b)+(f \varphi c d)$ is not a simple two-dimensional vector, but a compound element (zusammengesetzte Grösse). If this sum, however, is multiplied by a one-dimensional vector in either the $f$ notation or the $\varphi$ notation the result is a one-dimensional vector. Compound elements, as well as simple elements, can be used as operators to convert vectors or operators into other vectors or operators.

4. Vectors and vector invariants of the second order. The forms mentioned so far have involved only the first derivatives of the vector $f$. We call them vectors or invariants of the first order. Vectors or vector uperators which involve second derivatives of $f$ (or any of its equivalents) will be called vectors or vector operators of the second order.

The vectors $f_{i j}$ are as a rule not tangent to the space defined by $f$; thus if this space is contained in a euclidean space of $r$ dimensions we may write

$$
f=\sum_{i=1}^{i=r} x^{i} e^{i}
$$

where the $e$ 's are independent vectors of the containing space and the $x$ 's are functions of the parameters $u$ of the space defined by $f$. The $f_{i j}$ will then certainly lie in this larger space.

It is evident that the $f_{i j}$ are not completely defined by the differential form $\sum E_{i j} d u_{i} d u_{j}$; in fact $f$ itself is not completely determined, since it may be the defining vector of any of the surfaces applicable to one another each having the same first differential form.

There are, however, certain restrictions on the vectors $f_{i j}$. From the equation $f_{i} f_{j}=E_{i j}$, we obtain by differentiation $f_{i} f_{j k}+f_{i k} f_{j}=\partial E_{i j} / \partial u_{k}$ and from this we obtain Maschke's formulas for the Christoffel triple index symbols

$$
f_{i} f_{k m}=\frac{1}{2}\left[\frac{\partial E_{i k}}{\partial u_{m}}+\frac{\partial E_{i m}}{\partial \iota_{k}}-\frac{\partial E_{k m}}{\partial u_{i}}\right] \text {. }
$$


Thus the scalar products of the tangent vectors $f_{i}$ and the vectors $f_{k m}$ are completely determined by the differential form (1). Maschke has shown that the combinations $f_{i r} f_{k s}-f_{k r} f_{i s}$ are also completely determined. For ordinary space the various restrictions on the second derivatives of the defining vector $f$ are all summed in the well known relations of Gauss and Codazzi. The corresponding relations for spaces in general will be given later.

The tangent $n$-dimensional vector $\left(f^{1}, \cdots, f^{n}\right)$, where $f^{1}, \cdots, f^{n}$ are all equivalent symbols of the differential form (1), is an invariant of the first order. The derivatives of this vector with respect to the parameters $u_{i}$ will all be expressions of the second order. They are not, however, in general simple elements but compound elements in a space of more than $n$ dimensions.

5. Normal vectors. Although the vectors $f_{i j}$ do not, in general, lie in the tangent space to the space $f$ they are, as a rule, not normal. It can be shown, however, that the first normals to all curves lying in $f$ are expressible in terms of the tangent vectors $f_{i}$ and the vectors $f_{i j}$. Any normal to the surface that can be expressed in terms of the first and second derivatives of $f$ will be called a first normal. If the $f_{i j}$ are linearly independent there are $n(n+1) / 2$ linearly independent first normals. One possible choice of these is the set

$$
f_{i j}-\sum\left\{\begin{array}{c}
i j \\
k
\end{array}\right\} f_{k}=N_{i j}
$$

where $\left\{\begin{array}{c}i j \\ k\end{array}\right\}$ are Christoffel's triple index symbols of the second kind belonging to the differential form (1).

Other normal vectors to a two-dimensional surface $f$ can be obtained from the forms $(f \varphi)_{1}$ and $(f \varphi)_{2}$. These forms, as previously mentioned, are compound elements but when applied as operators to the vectors $\varphi_{1}$ and $\varphi_{2}$ they give expressions which are linear in the $f_{i j}$ and the $f_{k}$. We write

(3) $\quad a=\varphi_{1}(f \varphi)_{1}, \quad b=\varphi_{2}(f \varphi)_{2}, \quad c=\varphi_{1}(f \varphi)_{2}, \quad d=\varphi_{2}(f \varphi)_{1} .^{*}$

It is easily verified that each of these vectors is orthogonal to each of the tangent vectors $f_{1}$ and $f_{2}$.

The following vectors, written in invariant form, are all normal to the

\footnotetext{
* Black face type will be used for letters indicating vectors except for the vector $f$ and its derivatives in any of its equivalent notations.
} 
surface $f$ because they are linearly expressible in terms of the vectors $a, b, c, d$ given above:

$$
\begin{array}{ll}
(\varphi a)((f \varphi) a), & (\psi \varphi)(\psi a)((f \varphi) a), \\
(\varphi a)(\psi a)((f \varphi) \psi), & (\psi \varphi)(\psi(f \varphi)) .
\end{array}
$$

The last of these vanishes identically* and this gives the linear relation $E b-F(c+d)+G a=0$ which connects the normal vectors $a, b, c, d$.

In $n$ dimensions we have the formula $\left(f, \varphi^{1}, \cdots, \varphi^{n-1}\right)^{2}=n$ !, or, more briefly, $(f \varphi)^{2}=n !$; and consequently $(f \varphi)(f \varphi)_{i}=0$.

This shows that the compound $n$-dimensional vectors $(f \varphi)_{i}$ are orthogonal to the $n$-dimensional (simple) tangent vector $(f \varphi)$.

As in the two-dimensional case we have the normal vectors

$$
a_{i j}=\Phi_{i}(f \varphi)_{j},
$$

where $\Phi_{i}$ is the cofactor of $f_{i}$ in the determinant

$$
\left\{f, \varphi^{1} \cdots \varphi^{n-1}\right\} .
$$

That these are really orthogonal to the tangent vectors is easily seen by means of Maschke's formulat

$$
f_{k}(f \varphi)_{i}(a \varphi)=0,
$$

where $a$ is an arbitrary function; for example, if $a$ is taken to be the independent parameter $u_{j},(a \varphi)$ reduces to $\beta \varphi_{j}$ and the formula shows that $\beta f_{k} \cdot a_{j i}=0$.

Maschke's formula (52) referred to in the foot note below may be written in the form

$$
(\psi \varphi)\left(\psi,(f \varphi), a^{3}, \cdots, a^{n}\right)=0,
$$

where $a^{3}, \cdots, a^{n}$ are arbitrary functions of the parameters $u$.

6. The equations of Gauss and Codazzi. The scalar products of the first derivatives of the vector $f$ are the first fundamental quantities. Similarly the scalar products of the second derivatives could be taken as the second fundamental quantities. It seems more convenient, however, to use scalar products of the normal vectors as defined in equations (2), p. 579. We write $N_{i j} N_{r s}=L_{i j r s}$.

* See Maschke, Invariants, loc. cit., p. 455, formula (52).

$\dagger$ Invariants, loc. cit., formula (33). 
The equations (2) may be written

$$
\begin{aligned}
& f_{i j}=\sum_{r}\left\{\begin{array}{c}
i j \\
r
\end{array}\right\} f_{r}+N_{i j}, \\
& f_{i k}=\sum_{r}\left\{\begin{array}{c}
i k \\
r
\end{array}\right\} f_{r}+N_{i k} .
\end{aligned}
$$

Differentiating the first with respect to $u_{k}$ and the second with respect to $u_{j}$ and equating the results we obtain

$$
\sum_{r}\left\{\begin{array}{c}
i j \\
r
\end{array}\right\} f_{r k}+\sum_{r}\left\{\begin{array}{c}
i j \\
r
\end{array}\right\}_{k} f_{r}+\left[N_{i j}\right]_{k}=\sum_{r}\left\{\begin{array}{c}
i k \\
r
\end{array}\right\} f_{r j}+\sum_{r}\left\{\begin{array}{c}
i k \\
r\}_{j}
\end{array} f_{r}+\left[N_{i k}\right]_{j} .\right.
$$

From this equation we obtain, after multiplying by $f_{m}$ and transposing,

$$
\begin{gathered}
\sum_{r}\left\{\begin{array}{c}
i j \\
r
\end{array}\right\}\left[\begin{array}{c}
r k \\
m
\end{array}\right]-\sum_{r}\left\{\begin{array}{c}
i k \\
r
\end{array}\right\}\left[\begin{array}{c}
r j \\
m
\end{array}\right]+\sum_{r} E_{r m}\left[\begin{array}{c}
i j \\
r
\end{array}\right\}_{k}-\left\{\begin{array}{c}
i k\}_{j} \\
r
\end{array}\right] \\
=f_{m}\left[N_{i k}\right]_{j}-f_{m}\left[N_{i j}\right]_{k} .
\end{gathered}
$$

Now if the first of equations (4) is multiplied by $N_{m l k}$ it is found that $N_{m k} N_{i j}=N_{m k} f_{i j}=L_{i j m k}$; also from the equation $N_{i j} f_{m}=0$ we obtain by differentiation

$$
N_{i j} f_{m k}+f_{m}\left[N_{i j}\right]_{k}=0 \text {. }
$$

Using these equations the right hand side of (6) reduces to

$$
L_{i j m k}-L_{i k m j}
$$

Equation (6) then shows that the difference $L_{i j m k}-L_{i k m j}$ is a function of the first fundamental quantities and their derivatives. This is the generalization of Gauss's equation.

The second fundamental quantities $L$ must also satisfy a second set of equations which are the generalization of the Codazzi equations of ordinary surface theory. These equations, however, involve still other quantities besides the first and second fundamental quantities. The equations are obtained by multiplying both sides of (5) by the vector $N_{m s}$. The products $N_{m s} N_{i j k}$ will be denoted by $\left(\begin{array}{l}i j k \\ m s\end{array}\right)$. We obtain, then,

$$
\sum_{r}\left\{\begin{array}{c}
i j \\
r
\end{array}\right\} L_{r k m s}-\sum_{r}\left\{\begin{array}{c}
i k \\
r
\end{array}\right\} L_{r j m s}=\left(\begin{array}{c}
i k j \\
m s
\end{array}\right)-\left(\begin{array}{c}
i j k \\
m s
\end{array}\right) \text {. }
$$


The difference $\left(\begin{array}{c}i k j \\ m s\end{array}\right)-\left(\begin{array}{c}i j k \\ m s\end{array}\right)$ cannot be expressed independently of the relations just found, in terms of the $L$ 's and the $E_{i j}$, without further limitation on the space $f$. If, for example, the discussion is limited to spaces which have a single first normal the relatior ${ }_{1} \mathrm{~S}$ reduce to the usual Codazzi equations. Other forms of limitation on $f$ will lead to different forms of these integrability conditions.

7. Resolution of vectors in given directions. A tangent vector is resolved into its components in $n$ given directions tangent to the space in the usual manner; thus in a two-dimensional space consider the tangent vector

$$
(f \varphi)((\varphi a) a)
$$

To resolve this vector along the tangents to the curves $b=$ const. and $c=$ const. we write

$$
(f \varphi)((\rho a) a) .=l(f b)+m(f c) .
$$

By multiplying in turn by $(f b)$ and $(f c)$ two equations are obtained from which $l$ and $m$ can be determined:

$$
\begin{aligned}
& (f b)(f \varphi)((\varphi a) a)=l(f b)^{2}+m(f b)(f c), \\
& (f c)(f \varphi)((\varphi a) a)=l(f b)(f c)+m(f c)^{2} .
\end{aligned}
$$

If the determinant of the coefficients of $l$ and $m$ in these equations is zero the two directions $(f b)$ and $(f c)$ are not independent. But when this is not the case the equations can be solved for $l$ and $m$.

The values of $l$ and $m$ are evidently differential parameters and their geometric meanings are easily seen from the manner in which they are derived.

If $(f b)$ and $(f c)$ are mutually orthogonal, the expressions for $l$ and $m$ become quite simple since, in this case, $(f b)(f c)=0$. The expression for $(f \varphi)((\varphi a) a)$ then becomes

$$
(f \varphi)((\varphi a) a)=\frac{(\psi b)(\psi \varphi)((\varphi a) a)}{(\psi b)^{2}}\left(f^{\prime} b\right)+\frac{\left(\psi^{\prime \prime} c\right)(\psi \varphi)((\varphi a) a)}{(\psi c)^{2}}(f c) .
$$

These results and formulas are easily extended to $n$ dimensions.

Another method which will frequently be used is the following. Let $(f a b)$ be a tangent vector in a three-dimensional space.* The product $(f a b)(p q r)$, where $p, q$, and $r$ are scalar functions, has the same direction as $(f a b)$. This product can be written in several different ways as a sum

* The method may be extended in an obvious way to vectors in $n$ dimensions. 
of three such products. Any one element of either factor may be exchanged with each element of the other factor. The sum of the resulting products is equivalent to the original product; thus,

$$
(f a b)(p q r)=(f a p)(b q r)+(f p b)(a q r)+(p a b)(f q r) .
$$

In this way, the vector $(f a b)$ is resolved into three components along $(f a p),(f p b)$, and $(f q r)$. In case the functions $a, b, p, q, r$ are not independent in sets of three, some of the terms on the right vanish and no resolution of the vector $(f a b)$ is effected; for example, if $a$ is a multiple of $p$ the first and last terms vanish and the remaining one is a multiple of $(f a b)$.

Still another method which is sometimes useful for second order vectors in two dimensions depends on the identity

$$
((a b) c)+((b c) a)+((c a) b)=0 .
$$

By means of this the vector $((f a) b)$ can be expressed in terms of the vectors $((f b) a)$ and $((a b) f)$. This is not immediately applicable to higher dimensions but the other methods mentioned in this article can be used in any number of dimensions.

8. Derivatives with respect to arc length. In all the work that follows it will be convenient to be able to write down in invariant form the derivative of any function of the coördinates of a given space with respect to arc length along any curve.

Consider first an arbitrary two-dimensional surface defined by the vector $f(u, v)$ and let $a=$ const. define a curve on this surface.

Let $V$ be any vector or scalar function of $u$ and $v$.

For values of $u$ and $v$ along the curve $a, V$ is a function of a single variable, since $u$ and $v$ satisfy the equation $a=$ const.

Denoting arc length by $s$ we have

But

$$
\frac{d V}{d s}=V_{1} \frac{d u}{d s}+V_{\mathbf{2}} \frac{d v}{d s} .
$$

$$
a_{1} \frac{d u}{d s}+a_{2} \frac{d v}{d s}=0
$$

and

$$
\begin{aligned}
\frac{d u}{d s} & =-\frac{d u}{\sqrt{\left(E d u^{2}+2 F d u d v+G d v\right)^{2}}} \\
& =\frac{a_{2}}{\sqrt{\left(E a_{2}^{2}-2 F a_{1} a_{2}+G a_{1}^{2}\right)}}=\frac{\beta a_{2}}{\sqrt{\Delta_{1} a}},
\end{aligned}
$$

where $\Delta_{1} a$ is the well known first differential parameter. 
Hence, by substitution in (9) we obtain

$$
\frac{d V}{d s}=\frac{1}{a_{1}} \frac{d u}{d s}\left[V_{1} a_{2}-V_{2} a_{1}\right]=\frac{(V a)}{\sqrt{\Delta_{1} a}} .
$$

Thus, whether $V$ represents a vector or a scalar function of $u$ and $v$ we obtain $d V / d s$ by simply forming the invariant $(V a) / \sqrt{\Delta_{1} a}$.

This result can be generalized in the following way. Consider a space $f$ of $n$ dimensions and let $\boldsymbol{r}=\sum a^{i} f_{i}$ be any vector function of the coordinates tangent to $f$. The length of this vector is $\sqrt{\sum E_{i j} a^{i} a^{j}}=\sqrt{\mathfrak{r}^{2}}$.

If $V$ is any function of the coordinates we have for the derivative of $V$ with respect to arc length in any direction

$$
\frac{d V}{d s}=\sum V_{i} \frac{d u_{i}}{d s}
$$

But for any curve tangent to the vector $\mathfrak{r}$

$$
\frac{d u_{i}}{d s}=\lambda a_{i}
$$

where $\lambda$ is a proportionality factor, the same for every $i$. This factor is obtained from the equation

whence

$$
\sum E_{i j} \frac{d u_{i}}{d s} \frac{d u_{j}}{d s}=1=\lambda^{2} \sum E_{i j} a^{i} a^{j},
$$

$$
\lambda^{2}=\frac{1}{\mathfrak{r}^{2}}
$$

If we substitute these results in the expression for $d V / d s$ we obtain

$$
\frac{d V}{d s}=\frac{\sum a^{i}\left(\partial V / \partial u_{i}\right)}{\sqrt{\sum E_{i j} a^{i} a^{j}}}
$$

It follows that if $\mathfrak{r}$ is any vector tangent to the space $f$ and expressed in the $f$ notation, the derivative of any function $V$ with respect to the arc length in the direction $\mathfrak{r}$ is obtained from the expression for $\mathfrak{r}$ by replacing $f_{i}$ by $V_{i}(i=1,2, \cdots, n)$ and dividing the result by the length of $\boldsymbol{r}$.

As an example we write down the value of $d V / d \sigma$ where $\sigma$ denotes arc length along an orthogonal trajectory of $a=$ const. on a two-dimensional surface. In this case $\mathfrak{r}=(f \varphi)(\varphi a)$ and

$$
\frac{d V}{d \sigma}=\frac{(V \varphi)(\varphi a)}{\sqrt{\Delta_{1} a}}
$$


In applications the denominator in these formulas can often be omitted. For example from the equation

$$
(f a)(f b)=0
$$

which holds for two orthogonal families of curves in a two-dimensional manifold, we obtain by "differentiation"

$$
(f a)((f b) c)+((f a) c)(f b)=0 .
$$

Here the differentiation is performed with respect to arc length along the curve $c=$ const. and the denominator of the formula is omitted.

II. Two-dimensional SURfaces

9. Sequence of curvatures for any curve on a surface. We may apply the formulas given in the paper Functional differential geometry* for the normals and curvatures of any curve to find expressions for the normals and curvatures of any curve lying in any space.

For curves lying in a two-dimensional surface we have

$$
\begin{aligned}
& n_{0}=t=\frac{(f a)}{\sqrt{(f a)^{2}}}, \\
& \frac{n_{1}}{r_{1}}=\frac{(t a)}{\sqrt{(f a)^{2}}}, \\
& \cdot \cdot \cdot \cdot \cdot \cdot \cdot \cdot \cdot \cdot \cdot \\
& \frac{n_{j}}{r_{j}}=\frac{\left(n_{j-1} a\right)}{\sqrt{(f a)^{2}}}+\frac{n_{j-2}}{r_{j-1}},
\end{aligned}
$$

where $a=$ const. is the equation of the curve.

When these results are expressed in terms of the vector $f$ and its derivatives they furnish a perfectly definite method for obtaining in succession the required normals and curvatures.

For the principal normal (first normal), we have

$$
\frac{n_{1}}{r_{1}}=\frac{((f a) a)}{(\varphi a)^{2}}-\frac{(\varphi a)((\varphi a) a)}{\left[(\varphi a)^{2}\right]^{2}}(f a),
$$

and from this we obtain

$$
\frac{1}{r_{1}^{2}}=\frac{-[(\varphi a)((\varphi a) a)]^{2}+(\varphi a)^{2}((f a) a)^{2}}{\left[(\varphi a)^{2}\right]^{3}} .
$$

* Loc. cit., p. 321. 
This formula for the square of the first curvature of any curve on the surface $f$ contains in the denominator and in the first term of the numerator only the quantities $a, E, F, G$, and their derivatives. In the second term of the numerator, however, there occur certain of the products $f_{i j} f_{r s}$ which are second order quantities not expressible in terms of the $E, F, G$. These we regard as known.

If the expression for $n_{1}$ is substituted in the formula for $n_{2} / r_{2}$ it is seen that

$$
n_{2} / r_{2}=p(f a)+q((f a) a)+r(((f a) a) a),
$$

where $p, q$, and $r$ are certain differential parameters. In like manner we have in general

$$
n_{j} / r_{j}=p_{1}(f a)+p_{2}((f a) a)+\cdots+p_{j+1}(\cdots(f a) \cdots a),
$$

where in the last term there are $j+1$ parentheses.

It is clear that the square of the second curvature will contain third order quantities, the square of the third curvature fourth order quantities, and so on.

10. Components of the curvature vectors. The tangent vector $(f a)$ can be written in several different forms, and from each of them the curvature vector of the curve $a=$ const. can be computed. This gives a variety of different expressions for the curvature vector.

If we obtain $d t / d s$ from $(f \varphi)(\psi \varphi)(\psi a)=t V \overline{(f a)^{2}}$, one of the terms of the result is normal to the surface and the remaining terms are tangent to the surface. Hence we have

$$
\frac{d t}{d s}=\frac{(\psi \varphi)(\psi a)}{\sqrt{(f a)^{2}}} \frac{((f \varphi) a)}{\sqrt{(f a)^{2}}}+\text { tangent vector. }
$$

The tangential component is also easily obtained from this form of $t$, but it is more easily simplified when derived from the form on the right of equation (12). We write

$$
((f a) a)=p(f a)+q(f \varphi)(\varphi a)+N .
$$

The second term is tangent to the surface but normal to the curve. Since $(f a)$ and $(f \varphi)(\varphi a)$ are orthogonal to each other we may multiply in turn by $(f a)$ and $(f \varphi)(\varphi a)$ and obtain

$$
p=\frac{((f a) a)(f a)}{(f a)^{2}}, \quad q=\frac{((f a) a)(f \varphi)(\varphi a)}{(f a)^{2}} .
$$


Thus the component of $((f a) a) /(f a)^{2}$ along the tangent vector $(f a)$ just cancels the second term on the right in equation (12). The curvature vector then takes the form

$$
\frac{d t}{d s}=\frac{(\psi \varphi)(\varphi a)((\psi a) a)}{\left[(f a)^{2}\right]^{2}}(f \theta)(\theta a)+\frac{(\psi \varphi)(\psi a)((f \varphi) a)}{\left(f^{\prime} a\right)^{2}} .
$$

It is evident that there should be no component in the direction of $(f a)$ since if the first curvature vector of the curve $a=$ const. is resolved into two components, one normal to the surface, and the other tangent to the surface, the tangential component is orthogonal to the curve.

If we denote the vector of unit length in the direction of $(f \varphi)(\varphi a)$ by $t$ and the normal component of $d t / d s$ by $\mathfrak{a}$, equation (13) becomes

$$
\frac{d t}{d s}=\Gamma_{1} a \mathfrak{t}+\mathfrak{a},
$$

where $\Gamma_{1} a$ is the important differential parameter

$$
\frac{(\psi \varphi)(\varphi a)((\psi a) a)}{\left[\sqrt{(f a)^{2}}\right]^{3}} .
$$

11. Other curvature vectors. Besides the vector $d t / d s$ already studied, there are three other important curvature vectors: $d \mathfrak{t} / d \sigma, d t / d \sigma$, and $d \mathbf{t} / d s$, where $\sigma$ denotes arc length along the orthogonal trajectories of the curves $a=$ const.

Proceeding as above for $d t / d s$ we obtain, after reduction, the following formulas:

$$
\begin{aligned}
& \frac{d t}{d s}=\frac{(\psi \varphi)(\varphi a)((\psi a) a) \mathbf{t}}{\left[\Delta_{1} a\right]^{3 / 2}}+\frac{(\psi \varphi)(\psi a)((f \varphi) a)}{\left[\Delta_{1} a\right]}, \\
& \frac{d \mathbf{t}}{d \sigma}=\frac{(f \varphi)(f a)(\theta a)((\varphi a) \theta) t}{\left[\Delta_{1} a\right]^{3 / 2}}+\frac{(\varphi a)(\theta a)((f \varphi) a)}{\left[\Delta_{1} a\right]}, \\
& \frac{d t}{d \sigma}=\frac{(\psi \varphi)(\varphi a)(\theta a)((\psi a) a) \mathbf{t}}{\left[\Delta_{1} a\right]^{3 / 2}}+\frac{(\psi \varphi)(\psi a)(\theta a)((f \varphi) \theta)}{\left[\Delta_{1} a\right]}, \\
& \frac{d \mathbf{t}}{d s}=\frac{(\varphi \psi)(\varphi a)((\psi a) a) t}{\left[\Delta_{1} a\right]^{3 / 2}}+\frac{(\varphi a)((f \varphi) a)}{\left[\Delta_{1} a\right]} .
\end{aligned}
$$

The formula for $d t / d s$ is rewritten here and $\Delta_{1} a$ is used instead of $(f a)^{2}$. This is the well known differential parameter of the first order of ordinary differential geometry.

The numerator of the expression for the normal component of $d t / d \sigma$ can be simplified by resolving it into components according to rule $\mathrm{A}$ of 
$\S 7$. In the products $((f \varphi) \theta)(\psi a)$ exchange the element $\theta$ with the elements $\psi$ and $a$. Introducing the other two factors, the result may be written

$$
\begin{aligned}
(\psi \varphi)(\theta a)((f \varphi) \theta)(\psi a) & =(\psi \varphi)(\theta a)[((f \varphi) \psi)(\theta a)+((f \varphi) a)(\psi \theta)] \\
& =(\psi \varphi)((f \varphi) \psi)(\theta a)^{2}+(\psi \varphi)(\psi \theta)(\theta a)((f \varphi) a) .
\end{aligned}
$$

The first of these terms vanishes identically and the other reduces to $(\varphi a)((f \varphi) a)$. It follows that the normal components of $d t / d \sigma$ and $d t / d s$ are the same.

Equations (15) can now be rewritten as follows:

$$
\begin{aligned}
\frac{d t}{d s}=\Gamma_{1} a \mathbf{t}+\mathfrak{a} ; & \frac{d t}{d \sigma}=-\Gamma_{\mathbf{2}} a \mathbf{t}+\mathbf{m} ; \\
\frac{d \mathbf{t}}{d \sigma}=\Gamma_{\mathbf{2}} a t+\mathfrak{b} ; & \frac{d \mathbf{t}}{d s}=\boldsymbol{\Gamma}_{1} a t+\mathbf{m},
\end{aligned}
$$

where $\mathfrak{a}$ and $\mathfrak{b}$ denote the normal components of the curvature vectors of the curve $a=$ const. and its orthogonal trajectory and $\boldsymbol{m}$ denotes the normal component of either of the cross curvatures.

The differential parameters

$$
r_{1} a=\frac{(\psi \varphi)(\varphi a)((\psi a) a)}{\left[\Delta_{1} a\right]}, \quad I_{2} a=\frac{\left(f^{\prime} \varphi\right)\left(f^{\prime} a\right)(\theta a)((\varphi a) \theta)}{\left[\Delta_{1} a\right]}
$$

are the geodesic curvatures of the curves $a=$ const. and of their orthogonal trajectories. The curves $a=$ const. are geodesics if $(\psi \varphi)(\varphi a)((\psi a) a)=0$.

12. Relations connecting normal vectors. The three normals $\mathfrak{a}, \mathfrak{b}, \mathfrak{m}$ are invariant under change of parameters. They depend, however, on the function $a$.

From the two vectors $\mathfrak{a}$ and $\mathfrak{b}$ a vector can be obtained which is an invariant proper; i. e. one that does not depend upon an arbitrary function $a$.

From the identity

$$
(\psi \varphi)((f \varphi) a)+((f \varphi) \psi)(\varphi a)=((f \varphi) \varphi)(\psi a)
$$

we have immediately

$$
\mathfrak{a}+\mathfrak{b}=\frac{((f \varphi) \varphi)(\psi a)^{2}}{\Delta_{1} a}=((f \varphi) \varphi),
$$


and this expression does not contain the function $a$. It follows that the sum of the normal components of the first curvature vectors of any pair of orthogonal curves through a point is the same as the corresponding sum for any other pair. The invariant vector $\mathfrak{a}+\mathfrak{b}$ will be denoted by $2 \boldsymbol{h}$.

We may also determine the connection between the normal components of the curvature vectors of two different systems of curves.

Any vector function tangent to the surface may be written in the form $p t+q t$ where $p$ and $q$ are functions of the coordinates. If $p^{2}+q^{2}=1$ the length of the corresponding vector at each point of the surface is unity.

The curvature vector of the curves defined by the vector $p t+q t$ $\left(p^{2}+q^{2}=1\right)$ may be written

or also in the form

$$
p \frac{(p t+q \mathbf{t}, a)}{\sqrt{(f a)^{2}}}+q \frac{(p t+q \mathrm{t}, \varphi)(\varphi a)}{\sqrt{(f a)^{2}}},
$$

$$
p \frac{d[p t+q \mathbf{t}]}{d s_{a}}+q \frac{d[p t+q \mathbf{t}]}{d \sigma_{a}},
$$

where $s_{a}$ denotes arc length along $a=$ const. and $\sigma_{a}$ means arc length along the orthogonal trajectories of the curves $a$.

When expanded the values of $\frac{d t}{d s_{a}}, \frac{d \mathbf{t}}{d s_{a}}, \frac{d t}{d \sigma_{a}}$, and $\frac{d \mathbf{t}}{d \sigma_{a}}$ from equations (16) may be substituted. The result reduces to

$$
\begin{gathered}
\frac{d[p t+q \mathfrak{t}]}{d s}=\left[-\frac{(q a)}{\sqrt{(f a)^{2}}}+\frac{(p \varphi)(\varphi a)}{\sqrt{(f a)^{2}}}-p \Gamma_{1}+q \Gamma_{2}\right](q t-p \mathfrak{t}) \\
+p^{2} \mathfrak{a}+2 p q \mathfrak{m}+q^{2} \mathfrak{b} .
\end{gathered}
$$

It is clear from this equation that the tangential component of the curvature of the curves tangent to $p t+q t$ has at each point the direction of the orthogonal vector $q t-p t$. The magnitude of this component, given by the coefficient in brackets, is the geodesic curvature of the system of curves determined by $p t+q t$.

The normal component is $\overline{\mathfrak{a}}=p^{2} \mathfrak{a}+2 p q \mathfrak{m}+q^{2} \mathfrak{b}$. If we write $p=\cos \theta$, $q=\sin \theta$, this becomes

$$
\mathfrak{a} \cos ^{2} \theta+2 \mathfrak{m} \sin \theta \cos \theta+\mathfrak{b} \sin ^{2} \theta .
$$

The derivative of the orthogonal unit vector, $q t-p t$, may also be computed; likewise the two cross curvatures. The results are 


$$
\begin{aligned}
& \frac{d[q t-p \mathbf{t}]}{d \sigma}=\left[-\frac{p a}{\sqrt{\Delta a}}-\frac{(q \varphi)(\varphi a)}{\sqrt{\Delta a}}+q \Gamma_{1}+p \Gamma_{2}\right](p t+q \mathbf{t}) \\
& +q^{2} \mathfrak{a}-2 p q \mathfrak{m}+p^{2} \mathfrak{b} \\
& \text { (19) } \frac{d[q t-p t]}{d s}=\left[\frac{(q a)}{\sqrt{\Delta a}}-\frac{(p \varphi)(\varphi a)}{\sqrt{\Delta a}}+p \Gamma_{1}-q \Gamma_{2}\right](p t+q \mathbf{t}) \\
& +p q \mathfrak{a}+\left(q^{2}-p^{2}\right) \mathfrak{m}-p q \mathfrak{b}, \\
& \frac{d[p t+q \mathbf{t}]}{d \sigma}=\left[\frac{(p a)}{\sqrt{\Delta a}}+\frac{(q \varphi)(\varphi a)}{\sqrt{\Delta a}}-q \Gamma_{1}-p \Gamma_{2}\right](q t-p \mathbf{t}) \\
& +p q \mathfrak{a}+\left(q^{\mathbf{2}}-p^{2}\right) \mathfrak{m}-p q \mathfrak{b} \text {. }
\end{aligned}
$$

From these equations we have

$$
\begin{aligned}
\overline{\mathfrak{a}} & =\mathfrak{a} \cos ^{2} \theta+2 \mathfrak{m} \sin \theta \cos \theta+\mathfrak{b} \sin ^{2} \theta, \\
\overline{\mathfrak{b}} & =\mathfrak{a} \sin ^{2} \theta-2 \mathfrak{m} \sin \theta \cos \theta+\mathfrak{b} \cos ^{2} \theta, \\
\overline{\mathfrak{m}} & =(\mathfrak{a}-\mathfrak{b}) \sin \theta \cos \theta+\mathfrak{m}\left(\sin ^{2} \theta-\cos ^{2} \theta\right),
\end{aligned}
$$

where $\overline{\mathfrak{a}}, \overline{\mathbf{b}}, \overline{\mathfrak{m}}$ for the new system of curves correspond to $\mathfrak{a}, \mathfrak{b}, \mathfrak{m}$ of the old.

Again, if we denote by $G_{1}$ the geodesic curvature of the curves having the tangent vectors $p t+q t$, and by $G_{2}$ the geodesic curvature of the curves having the tangent vectors $q t-p t$ we have the formulas

$$
\begin{aligned}
& G_{1}=-\frac{(q a)}{\sqrt{\Delta a}}+\frac{(p \varphi)(\varphi a)}{\sqrt{\Delta a}}-p \Gamma_{1}+q \Gamma_{2}, \\
& G_{2}=-\frac{(p a)}{\sqrt{\Delta a}}-\frac{(q \varphi)(\varphi a)}{\sqrt{\Delta a}}+q \Gamma_{1}+p \Gamma_{2} .
\end{aligned}
$$

13. Planar curvature. The derivative of the unit two-dimensional vector, tangent to the surface at a point of the curve, taken with respect to arc length along this curve, is connected with the curvature of the curve itself and also with the well known Gaussian curvature of the surface. We shall call this quantity the planar curvature of the curve with respect to the surface $f$.

We have seen that the expression $(f \varphi)$ represents a two-dimensional vector tangent to the surface, and that $(f \varphi)^{2}=2$ !; hence, $\sqrt{\frac{1}{2}}(f \varphi)$ is the unit tangent two-dimensional vector.

Denoting the planar curvature vector by $k_{p}$ we have

$$
k_{p}=\frac{((f \varphi) a)}{\sqrt{2} \sqrt{\Delta_{1} a}}
$$


The magnitude of this vector is the planar curvaturve itself. Denoting it by $k$ we have,

$$
k^{2}=\frac{1}{2 \Delta_{1} a}((f \varphi) a)((f \varphi) a)
$$

The connection of this with the curvature vectors previously studied, and the Gaussian curvature, is found by multiplying by $(\psi \theta)^{2}$ with the use of rules $A$ and $B$ :

$$
\begin{aligned}
4 k^{2} \Delta_{1} a & =((f \varphi) a)(\psi \theta)((f \varphi) a)(\psi \theta) \\
& =[((f \varphi) \psi)(a \theta)+((f \varphi) \theta)(\psi a)]((f \varphi) a)(\psi \theta) \\
& =2((f \varphi) \psi)((f \varphi) a)(a \theta)(\psi \theta) \\
& =2[((f \psi) \varphi)+((\psi \varphi) f)]((f \varphi) a)(\psi \theta)(a \theta) \\
& =4((f \psi) \varphi)((f \varphi) a)(\psi \theta)(a \theta) \\
& =4((f \psi) \psi)(\varphi \theta)(a \theta)((f \varphi) a)+4((f \psi) \theta)(\psi \varphi)((f \varphi) a)(a \theta) \text { (Rule A). }
\end{aligned}
$$

The first term of this expression is evidently equivalent to

$$
4(\mathfrak{a}+\mathfrak{b}) \mathfrak{a}(\theta a)^{2} .
$$

The second term can be reduced as follows:

$((f \psi) \theta)(\psi \varphi)((f \varphi) a)(a \theta)=((f \psi) a)((f \varphi) \theta)(\psi \varphi)(a \theta)-((f \psi)(f \varphi))(\psi \varphi)(a \theta)^{2}$.

The first term on the right is the negative of the term on the left and the second term on the right contains the factor $((f \psi)(f \varphi))(\psi \varphi)$, which Maschke has shown to be twice the Gaussian curvature.

Substituting these results into the expression for $4 \Delta_{1} a k^{2}$ and dividing through by $\Delta_{1} a=(\theta a)^{2}$ we have the result

$$
k^{2}=\mathfrak{a}(\mathfrak{a}+\mathfrak{b})-K
$$

The square of the planar curvature in the orthogonal direction is clearly $\mathfrak{b}(\mathfrak{a}+\mathfrak{b})-K$ and the sum of the squares of the curvatures in the two orthogonal directions is therefore equal to $4 h^{2}-2 K$ which is independent of the curve $a=$ const.; later it will be seen that this invariant may be written $\frac{1}{2}((f \varphi) \psi)((f \varphi) \psi)$.

14. Principal directions for planar curvature. The expression for

$$
k^{2}=\frac{1}{2} \frac{\overline{d(f \varphi)^{2}}}{d s^{2}}
$$


can be put in another useful form; $d(f \varphi)$ can be written $d(f \varphi)=(f \varphi)_{1} d u$ $+(f \varphi)_{2} d v$ and from this we obtain

where

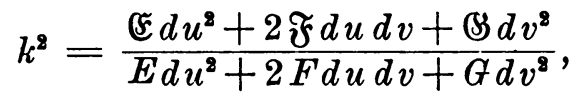

$$
\mathfrak{F}=\frac{1}{2}(f \varphi)_{1}^{2}, \quad \mathfrak{F}=\frac{1}{2}(f \varphi)_{1}(f \varphi)_{2}, \quad \text { and } \quad \mathscr{S}=\frac{1}{2}(f \varphi)_{2}^{2} .
$$

These quantities can evidently be expressed in terms of any of the sets which have been referred to as second fundamental quantities.

The values of $\lambda=d v / d u$ which make $k^{2}$ a maximum or minimum are found, in the usual manner, to satisfy the equation

$$
(E \mathfrak{F}-\mathbb{F} F)+\left(E \mathscr{S}-(\mathfrak{F} G) \lambda+(F \mathbb{S}-\mathfrak{F} G) \lambda^{2}=0\right.
$$

The corresponding values of $R=1 / k^{2}$, also found in the usual way, are given by the equation

$$
\left(\mathfrak{S}-\mathfrak{F}^{2}\right) R^{2}-\left(G(\mathfrak{F}-2 F \mathfrak{F}+E \mathbb{S}) R+E G-F^{2}=0 .\right.
$$

If $a=$ const. is the equation of a curve whose direction at each point coincides with the direction determined by one of the values of $\lambda$ obtained from the above equation, then $a$ satisfies the differential equation

$$
(f(\varphi \psi))(f a)((\varphi \psi) a)=0 .
$$

This equation may be reduced as follows:

$$
\begin{aligned}
(f(\varphi \psi))(f a)((\varphi \psi) a) & =(\varphi(f \psi))(f a)((\varphi \psi) a)+(\psi(\varphi f))(f a)((\varphi \psi) a) \\
& =2(\varphi(f \psi))(f a)((\varphi \psi) a) \\
& =2(f(f \psi))(\varphi a)((\varphi \psi) a)+2(a(f \psi))(f \varphi)((\varphi \psi) a) .
\end{aligned}
$$

The second term of this last expression vanishes so that the equation reduces to

or

$$
(f(f \psi))(\varphi a)((\varphi \psi) a)=0
$$

$$
\boldsymbol{h} \cdot \mathbf{m}=0 \text {. }
$$

The two directions defined by the equation in $\lambda$ are orthogonal as may be seen by applying the test usually given in ordinary differential geometry, which is equally valid here. 
In the equation for $R$ put $f_{1}^{2}, f_{2}^{2}$, and $f_{1} f_{2}$ for $E, F$, and $G$, respertively,

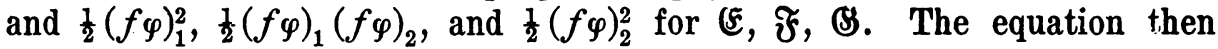
becomes

$$
\frac{1}{2} \beta(\theta \psi)_{1}(f \varphi)_{2}((\theta \psi)(f \varphi)) R^{2}-\frac{1}{2}(f(\varphi \psi))^{y} R+1=0 .
$$

The coefficient of $R^{2}$ may also be written

$$
-\beta(f \varphi)_{1}(\theta \psi)_{2}((\theta \psi)(f \varphi)),
$$

as is seen by interchanging $f$ with $\theta$ and $\psi$ with $\varphi$. Thus the coefficient of $R^{2}$ may finally be written

$$
\frac{1}{2}((\theta \psi)(f \varphi))^{2},
$$

and the equation for $R$ becomes

$$
\frac{1}{4}((\theta \psi)(f \varphi))^{2} R^{2}-\frac{1}{2}(f(\varphi \psi))^{2} R+1=0 .
$$

For surfaces in ordinary space the directions for the maximum and minimum of $[d(f \varphi) / d s]^{2}$ coincide with the principal directions and

$$
\frac{\mathfrak{E} B-\mathfrak{F}^{2}}{E G-F^{2}}=K
$$

15. Various differential parameters and invariants. It is possible to write down immediately, in the symbolic notations, a number of invariants. Some of these will involve only the first fundamental quantities and their derivatives. Others will involve the second fundamental quantities as well. In two dimensions the only invariant of the first of these types is the Gaussian curvature, $K$. Maschke has given the formula

The invariants

$$
K=\frac{1}{2}((\varphi f)(\psi f))(\varphi \psi) .
$$

$$
((f \varphi) \varphi)((f \psi) \psi)=4 h^{2}, \quad \text { and } \quad((f \varphi) \psi)((f \varphi) \psi)
$$

are examples of invariants which depend upon the second as well as the first fundamental quantities.

Various scalar products formed from the vectors $\mathfrak{a}, \mathfrak{b}, \mathfrak{m}$ furnish examples of differential parameters of the second order depending on one arbitrary function $a$ of the coordinates. 
There exist a number of interesting relations connecting these invariants and differential parameters and some of these will now be derived.

(a) The invariant

may be written

$$
((f \varphi) \psi)((f \varphi) \psi)
$$

$$
\begin{aligned}
{[((f \psi) \varphi)+((\psi \varphi) f)]((f \varphi) \psi) } & =2((f \psi) \varphi)((f \varphi) \psi) \\
& =2((f \psi)(f \varphi))(\varphi \psi)+2((f \psi) \psi)((f \varphi) \varphi) \\
& =-4 K+8 h^{2} .
\end{aligned}
$$

(b) From formulas (15) we have

$$
\begin{aligned}
& \frac{1}{\Delta_{1} a^{2}} \mathfrak{a} \cdot \mathfrak{b}=(\psi \varphi)(\psi a)((f \varphi) a)(\varrho a)(p a)((f \varrho) \theta) \\
& =(\psi \varphi)(\theta a) .((f \varphi) a)(\varrho a)((f \varrho) \psi)(\theta a)+(\psi \varphi)(\theta a)((f \varphi) a)(\varrho a)((f \varrho) a)(\psi \theta) .
\end{aligned}
$$

The last term reduces to $\left(\Delta_{1} a\right)^{2} \mathbf{m}^{2}$, since $(\psi \varphi)(\psi \theta)(\theta a)=(\varphi a)$. The first term can be reduced by similar methods to $\left(\Delta_{1} a\right)^{2} K$. Thus we obtain the formula

or

$$
\left(\Delta_{1} a\right)^{\mathbf{a}} \mathfrak{a} \cdot \mathfrak{b}=\left(\Delta_{1} a\right)^{\mathbf{2}} K+\left(\Delta_{1} a\right)^{2} \mathfrak{m}^{2},
$$

$$
K=\mathfrak{a} \cdot \mathfrak{b}-\mathfrak{m}^{2} .
$$

III. Geometry of $n$-Dimensional manifoldS

16. Curvatures of an orthogonal system of curves. We consider now an arbitrary $n$-dimensional space defined by the vector $f\left(u_{1}, u_{2}, u_{3}, \cdots, u_{n}\right)$.

Let $n$ subspaces each of $n-1$ dimensions mutually orthogonal at a point $P$ be defined by the system of equations

$$
a^{1}=\text { const., } \quad a^{2}=\text { const., } \cdots, a^{n}=\text { const. }
$$

Any system of $n-1$ of the equations of the system (22) defines a curve in the space $f$. The tangent vectors to these curves are

$$
\left(f a^{1} a^{2} \cdots a^{i-1} a^{i+1} \cdots a^{n}\right),
$$

and these vectors are mutually orthogonal.* We write

$$
t^{i}=\frac{(f a)}{\sqrt{(f a)^{2}}}
$$

where $(f a)$ is an abbreviation for $\left(f a^{1} a^{2} \cdots a^{i-1} a^{i+1} \cdots a^{n}\right)$.

\footnotetext{
* It will be understood that the discussion relates to the point $P$ mentioned above.
} 
The unit tangent vector $t^{i}$ may also be written in the form

$$
t^{i}=\frac{(f \varphi)(\psi \varphi)(\psi a)}{(n-1) ! \sqrt{(f a)^{2}}}
$$

It will be convenient to denote $(f a)^{2}$ by $\Delta a$. Using this abbreviation we write down at once, by formula (11), the expression for $d t^{i} / d s_{i}$ :

$$
\begin{aligned}
\frac{d t^{i}}{d s_{i}}=\frac{(f \varphi)(\psi \varphi)((\psi a) a)}{(n-1) ! \Delta a} & +\frac{(\psi \varphi)(\psi a)((f \varphi) a)}{(n-1) ! \Delta a} \\
& -\frac{(\psi a)((\psi a) a)}{(n-1) !(\Delta a)}(f \varphi)(\theta \varphi)(\theta a) .^{*}
\end{aligned}
$$

The first term of this expression is tangent to the space $f$ and may be expressed linearly in terms of the vectors $(f c)$ where $c$ represents the various combinations of $n-1$ of the functions $a$. One of the terms of this expansion (the one for which the corresponding combination $c$ has $a^{i}$ missing) will just cancel the negative term at the end and we have the result

$$
\frac{d t^{i}}{d s_{i}}=\sum_{c} \frac{(\psi c)((\psi a) a)}{\Delta c \Delta a}(f c)+\frac{(\psi \varphi)(\psi a)((f \varphi) a)}{(n-1) ! \Delta a}
$$

where the mark (,) above the summation sign indicates that the term in which the combination $c$ is identical with the combination $a$ is to be omitted.

The fact that the term $(f a)$ is missing shows that the tangential component of the curvature vector $d t^{i} / d s_{i}$ is orthogonal to $t^{i}$. This is, of course, to be expected.

17. Relations among the normal components. The normal components

$$
\mathfrak{a}^{i}=\frac{(\psi \varphi)(\psi a)((f \varphi) a)}{(n-1) ! \Delta a}
$$

of the various curvature vectors $d t^{i} / d s_{i}$ can be modified in the following manner.

Omit the denominator temporarily and suppose that the combination $b$ is the one in which the function $a^{k}$ is missing, and $c$ is the combination in which $a^{j}$ is missing. We may write

$$
(\psi \varphi)(\psi b)((f \varphi) b)=\frac{(\psi \varphi)(\psi b)((f \varphi) b)(a)}{(a)}
$$

* The term containing the product $(f \varphi)(\psi a)((\psi \varphi) a)$ vanishes. See Maschke, Invariants, loc. cit., formula (33). 
where in the factor $(a)$, of course, all the $a$ 's are present. Now

$$
(\psi \varphi)(a)=\sum_{c}\left(a^{j} \varphi\right)(\psi c)=\sum_{j}\left(a^{j} \varphi\right)(a \psi a),
$$

where $\psi$ in the last factor occupies the $j$ th position.

If the terms of the sum on the right are multiplied by $(\psi b)$ they will all vanish except the one for which $j=k$ since the vectors $(\psi b)$ and $(\psi c)$ are orthogonal; thus,

$$
\begin{aligned}
\frac{(\psi \varphi)(\psi b)((f \varphi) b)}{(n-1) ! \Delta b} & =(-1)^{k-1} \frac{\left(a^{k} \varphi\right)(\psi b)^{2}((f \varphi) b)}{(n-1) ! \Delta b(a)} \\
& =(-1)^{k-1} \frac{\left(a^{k} \varphi\right)((f \varphi) b)}{(n-1) !(a)}=\mathfrak{a}^{k}
\end{aligned}
$$

This gives, then, a new expression for the normal components of the curvature vector $d t^{k} / d s_{k}$.

We now run the $a^{k}$ of the first factor through the last factor and obtain

$$
\mathbf{a}^{k}=\frac{((f \varphi) \varphi)}{(n-1) !}+\sum_{j}(-1)^{k-1} \frac{\left(a^{j} \varphi\right)\left((f \varphi) a a^{k} a\right)}{(n-1) !(a)},
$$

where in the last factor of the numerator the $a^{k}$ occupies the $j$ th position. To bring $a^{k}$ to its proper position we must pass it over $k-j-1$ letters if $k>j$ or over $j-k-1$ letters if $k<j$. The resulting effective power of $(-1)$ is in either case equal to $j$ so that the $j$ th term is the negative of $\mathfrak{a}^{j}$; hence we have

$$
\sum_{i} \mathfrak{a}^{i}=\frac{((f \varphi) \varphi)}{(n-1) !} .
$$

18. The cross curvatures. By a procedure similar to that of $\S 11$ we obtain the formulas for $d t^{i} / d s_{k}$.

Let $a$ denote the combination corresponding to $t^{i}$ and $b$ the combination corresponding to $t^{k}$; also let $c$ denote any combination. We have then

$$
\frac{d t^{i}}{d s_{k}}=\sum_{c}^{\prime} \frac{(\psi c)((\psi a) b)}{\Delta c \sqrt{\Delta a} \sqrt{\Delta b}}(f c)+\frac{(\psi \varphi)(\psi a)((f \varphi) b)}{(n-1) ! \sqrt{\Delta a} \sqrt{\Delta b}} .
$$

This formula exhibits the curvature vector $d t^{i} / d s_{k}$ as the sum of two components, one normal to the space $f$ and the other tangent to $f$. If $a$ and $b$ are interchanged we have the formula for $d t^{k} / d s_{i}$. We shall now show that the normal components of these two vectors are the same. 
Consider the special case in which $a$ is the combination $a^{2} \cdots a^{n}$ and $b$ is the combination $a^{1} a^{3} \cdots a^{n}$. In the product $(\psi a)((f \varphi) b)$ run the $a^{2}$ of the first factor through the second. The first factor of each term of the result, except the first two, will contain a repeated $a$, and we may write

$$
\begin{aligned}
(\psi \varphi)(\psi a)((f \varphi) b) & =(\psi \varphi)\left(\psi(f \varphi) a^{3} \cdots a^{n}\right)\left(a^{2} a^{1} a^{3} \cdots a^{n}\right) \\
& +(\psi \varphi)(\psi b)((f \varphi) a) .
\end{aligned}
$$

The first expression on the right vanishes* and the second is the result of interchanging $a$ and $b$ in the expression on the left. The two normals corresponding to these choices of the combinations $a$ and $b$ are thus seen to be the same. The same proof can be used for every choice of $a$ and $b$.

The expressions $(\psi c)((\psi a) b)$ which occur in the coefficients of $(f c)$ in the expression above for $d t^{i} / d s_{k}$ are important differential parameters. From the formula $(\psi c)(\psi a)=0$ we derive the equation

$$
(\psi c)((\psi a) b)+(\psi a)((\psi c) b)=0,
$$

which is an important relation connecting these quantities. These quantities are closely related to Ricci's coefficients of rotation between which there exist relations of precisely the same nature as the one just obtained.

19. Generalization of planar curvature. The generalized planar curvature in $n$ dimensions is defined in a manner altogether analogous to that used for the corresponding idea in two dimensions.

The tangent $n$-dimensional space may be written $(f)$ as in the twodimensional case, and its magnitude is determined from the relation

$$
(f)^{2}=n !
$$

The unit $n$-dimensional tangent vector is therefore

$$
T=\frac{1}{\sqrt{(n !)}}(f) .
$$

The derivative of $T$ with respect to arc length in any given direction may be taken as the (vector) planar curvature in that direction. The numerical value may, as usual, be computed from the scalar square of the vector curvature.

* See Maschke, loc. cit., formula 52, p. 455. 
If we write $\mathfrak{\xi}_{i j}=(1 / n !)(f)_{i}(f)_{j}$, we have at once

$$
\left[\frac{1}{\sqrt{n !}} \frac{d(f)}{d s}\right]^{2}=\frac{\sum_{j} \sum_{i} \S_{i j} d u_{i} d u_{j}}{\sum_{j} \sum_{i} E_{i j} d u_{i} d u_{j}}=\frac{1}{R} .
$$

If $d u_{1} \ldots d u_{n}$ are proportional to the variables $\lambda_{1} \ldots \lambda_{n}$ the necessary conditions for maximum or minimum values of $1 / R$ may be written

$$
\left(\sum_{j} \sum_{i} E_{i j} \lambda_{i} \lambda_{j}\right)\left(\sum_{i} \xi_{i k} \lambda_{i}\right)-\left(\sum_{j} \sum_{i} \xi_{i j} \lambda_{i} \lambda_{j}\right)\left(\sum_{i} E_{i k} \lambda_{i}\right)=0,
$$

and hence for these values of $R$

and hence also

$$
\frac{1}{R}=\frac{\sum_{i} \mathfrak{E}_{i k} \lambda_{i}}{\sum_{i} E_{i k} \lambda_{i}}
$$

$$
\sum_{i}\left(E_{i k}-R \xi_{i k}\right) \lambda_{i}=0 .
$$

The elimination of the variables $\lambda_{i}$ from these equations leads to an equation of the $n$th degree in $R$ which we write in the form

$$
1+J_{1} R+J_{2} R^{2}+\cdots+J_{n} R^{n}=0 .
$$

The coefficients $J$ can be expressed symbolically. Replace $E_{i k}$ and $\mathfrak{F}_{i k}$ by $f_{i} f_{k}$ and $(1 / n !)(f)_{i}(f)_{k}$ respectively, and reduce by the method of Bates (loc. cit., p. 27):

$$
J_{m}=\frac{1}{n !} \frac{1}{n ! m !(n-m) !}\left((f)^{1}(f)^{2} \cdots(f)^{m} f\right)^{2},
$$

where $(f)^{1} \cdots(f)^{m}$ each contain $n$ equivalent symbols distinct from one another and also distinct from the remaining $f^{\prime}$ s which fill out the invariantive constituent.

As in Bates, formula 29, p. 24, we may write equation (31) in the form

$$
f_{k} \sum_{i} f_{i} d u_{i}=R(f)_{k} \sum_{i}(f)_{i} d u_{i}
$$

and also in the form

$$
f_{k}(f a)=R(f)_{k}((f) a) .
$$

20. Principal directions. Lines of curvature. The directions which correspond to the stationary values of $R$, when they are all distinct, are the principal directions. A line of curvature is a curve whose direction at each point coincides with a principal direction. 
For two different lines of curvature

$$
\begin{aligned}
& \left(f^{\prime}\right)_{k}\left(\left(f^{\prime}\right) a\right)=\frac{1}{R_{1}} f_{k}(f a), \\
& \left(f^{\prime}\right)_{k}\left(\left(f^{\prime}\right) b\right)=\frac{1}{R_{2}} f_{k}(f b) .
\end{aligned}
$$

Now the cofactor of $(f)_{i}$ in the determinant $((f) a)$ is the same as the cofactor of $f_{i}$ in $(f a)$ since they both depend in the same way on the functions $a$; hence if we multiply the first of the above equations by the cofactors of $f_{k}$ in the determinant $(f b)$ and add the results for $k=1,2, \cdots, n$, we have

$$
((f) a)((f) b)=\frac{1}{R_{1}}(f a)(f b)
$$

and similarly from the second of the above

$$
((f) a)((f) b)=\frac{1}{R_{2}}(f a)(f b)
$$

hence if $R_{1}$ and $R_{2}$ are different we must have

$$
(f a)(f b)=0
$$

and the corresponding lines of curvature are orthogonal.

UNIVERSITY OF MISSOURI,

Columbia, Mo. 